\title{
Expression of the epigenetic H3K27me3 modifier genes KDM6A and EZH2 in patients with upper tract urothelial carcinoma
}

\author{
YUE WANG $^{1,2}$, JIN-XIA ZHANG $^{1}$, SHU-FANG WANG $^{1}$, YU ZHANG $^{1}$ and HUI-YING HE ${ }^{2}$ \\ ${ }^{1}$ Department of Pathology, Peking University Shougang Hospital, Beijing 100144; \\ ${ }^{2}$ Department of Pathology, School of Basic Medical Sciences, Peking University Third Hospital, \\ Peking University Health Science Center, Beijing 100083, P.R. China
}

Received May 18, 2020; Accepted September 11, 2020

DOI: $10.3892 / \mathrm{ol} .2020 .12212$

\begin{abstract}
The development of upper tract urothelial carcinoma (UTUC) has been associated with the ingestion of aristolochic acid (AA) in Chinese herbs. The tumors are more malignant and patients have a worse prognosis in China compared with that in Western countries. Recently, whole-genome and exome sequencing of AA-associated UTUCs found that the most frequently mutated gene was lysine demethylase 6A (KDM6A). However, its biological role and clinical significance have not yet been defined in patients with UTUC in China. A total of 108 surgically resected UTUC samples were obtained. Using immunohistochemistry, the protein expression level of KDM6A in the tumors was investigated together with the clinical and pathological characteristics of the patients, including survival times. In the present study, the expression level of KDM6A was significantly lower in UTUC specimens compared with that in samples from the normal urothelium. Lower KDM6A expression was also found to be significantly associated with a higher tumor grade and shorter cancer-specific and disease-free survival times $(\mathrm{P}=0.023$ and $\mathrm{P}=0.033$, respectively). In addition, using immunohistochemical analysis, no positive association was found between KDM6A expression and the expression of H3K27me3 or histone-lysine N-methyltransferase EZH2, a histone methyltransferase that generates $\mathrm{H} 3 \mathrm{~K} 27$ me3. The results of the present study indicated that decreased KDM6A expression level was significantly associated with tumor grade and decreased survival time in UTUC, suggesting that KDM6A expression could be used as a prognostic marker in patients with UTUC in China.
\end{abstract}

Correspondence to: Professor Hui-Ying He, Department of Pathology, School of Basic Medical Sciences, Peking University Third Hospital, Peking University Health Science Center, 38 College Road, Haidian, Beijing 100083, P.R. China

E-mail: huiyinghe@bjmu.edu.cn

Key words: upper tract urothelial carcinoma, KDM6A, EZH2, H3K27me3, prognosis

\section{Introduction}

Upper tract urothelial carcinoma (UTUC), derived from the urothelium of the renal pelvis and ureter, is relatively uncommon and comprises only $5-10 \%$ of all urothelial carcinoma cases in Western countries (1). However, in some regions of China there is a higher prevalence rate of UTUC, accounting for up to $20-30 \%$ of all urothelial carcinoma cases (2). Furthermore, compared with the USA, patients in China appear to be younger and exhibit worse prognostic features, including high histological grade, muscle invasion and nodal metastasis (2), suggesting that there may be specific genetic or environmental factors that influence UTUC carcinogenesis in the Chinese population. Aristolochic acid (AA), a constituent in numerous traditional Chinese herbs, may contribute to the higher incidence rate of UTUC in the Chinese population (2). This is corroborated by a histone demethylase gene, lysine demethylase 6A (KDM6A), which has been identified as the most frequently mutated gene in patients with UTUC in China (3).

$K D M 6 A$, also known as $U T X$, was the first mutated histone demethylase gene described in human cancer. The gene was initially demonstrated to be essential in the differentiation of the normal urothelium (4). Subsequently, somatic mutations and deletions targeting the KDM6A gene were identified in a variety of different types of human cancer, including multiple myeloma, bladder cancer and prostate cancer (5-7). KDM6A is the second most significantly altered cancer-associated gene in bladder cancer, and bladder cancer is the most frequent malignancy in tumors with the KDM6A mutation $(5,8)$, suggesting an important role for KDM6A in the tumorigenesis of urothelial carcinoma. However, the prognostic value of KDM6A expression in patients with UTUC has not yet been investigated. Previous studies have indicated that KDM6A functions, in part, by antagonizing histone-lysine $\mathrm{N}$-methyltransferase EZH2 (EZH2), which provides instructions for synthesizing histone methyltransferase through the removal of methyl groups from $\mathrm{H} 3 \mathrm{~K} 27 \mathrm{me} 3$, which provides an epigenetic modification of histone $\mathrm{H} 3(9,10)$. Bladder cancer with inactivating $K D M 6 A$ mutations is potentially targetable through the inhibition of EZH2 (4). To the best of our knowledge, this is the first study to analyze the expression levels of KDM6A, EZH2 and H3K27me3, and the interactions among these proteins in UTUC. 


\section{Materials and methods}

Patients. Tissues were retrieved from 108 patients with UTUC, who received radical nephroureterectomy between January 2007 and March 2017 at Peking University Shougang Hospital and Peking University Third Hospital (Beijing, China). Written informed consent was obtained from all patients. None of the patients received preoperative chemotherapy. All cases were reviewed, and pathological diagnoses were confirmed independently by two pathologists. The pathological grade was assigned according to the 2016 World Health Organization histological criteria (11), and the tumor stage was assigned using the American Joint Committee on Cancer TNM Staging System for Renal Pelvis and Ureter Cancer (8th edition, 2018) (12).

Patient follow-up data were collected until June 2017. However, 14 patients were lost to follow-up after surgery, leaving 94 cases for final survival analysis, with a median follow-up time of 28 months (range, 1-101 months). Bladder recurrence was defined as the identification of a subsequent bladder tumor during cystoscopy, with confirmation using a pathological evaluation. Cancer-specific survival (CSS) was defined as the interval between surgery and death from UTUC. Death was scored as an event, and patients who were still alive were contacted twice a year to assess the state of their health. Disease-free survival (DFS) was calculated from the date of surgery to the date of the first documented evidence of disease recurrence, or the last follow-up visit, while the patient was alive. Patient characteristics are shown in Table I.

Construction of tissue microarrays (TMAs). TMAs were constructed from 108 samples of UTUC, and 29 samples of adjacent normal urothelium. A power calculation was performed to support the inclusion of the number of samples. TMAs were constructed with the three representative areas of the tumor, which were selected manually. The representative areas were defined as the areas with enough tumor cells, less necrosis, no extrusion and heterogeneous regions if they exist. A previous study found that $\geq 3$ cores from each sample provides an acceptable statistical analysis in TMAs in various tumor types (13), and so three 1-mm tissue cores from each $10 \%$ neutral buffered formalin-fixed $(12-48 \mathrm{~h}$ at room temperature) paraffin-embedded donor block were selected and precisely arrayed into a new recipient paraffin block.

Immunohistochemistry (IHC) of TMA and scoring. A total of four serial 4-mm sections were prepared from each TMA section. The first slide was stained with hematoxylin $(5 \mathrm{~min})$ and eosin (1-3 $\mathrm{min})$ at room temperature for hematoxylin to confirm the presence of tumor cells, and subsequent slides were used to evaluate the reactivity of primary antibodies, including rabbit polyclonal anti-KDM6A antibody (1:100; cat. no. ab36938; Abcam), mouse monoclonal anti-EZH2 antibody (1:150; cat. no. 6A10; Origene Technologies, Inc.) and rabbit polyclonal anti-H3K27me3 antibody (1:150; cat. no. A2363; ABclonal Biotech Co., Ltd.). The Tris-buffered saline without antibodies were used as a negative control. Hydrogen peroxide (3\%) was used as blocking reagent at room temperature for $10 \mathrm{~min}$. Primary antibodies were all incubated for $4^{\circ} \mathrm{C}$ overnight. Secondary antibody for Pika (neat; cat. no. PV-6,000; Origene Technologies, Inc.) was used at room temperature for $30 \mathrm{~min}$. Immunostaining results were evaluated independently by two pathologists. Discrepancies in analysis were reconciled following evaluation by a third reviewer. Semi-quantitative scoring criteria, including staining intensity and proportion of positive cells, were used for KDM6A, EZH2 and H3K27me3 staining evaluation. The normal urothelium showed strong immunoreactivity for KDM6A, allowing its use as a positive internal control. Tonsil tissue and glioma cells served as positive controls for EZH2 and H3K27me3, respectively. These tissues and cells were obtained from Peking University Shougang Hospital (Beijing, China) (approval no. IRBK-2017-047-01). Written informed consent was obtained from all patients. Thus, staining intensity that was equivalent to a positive internal control was graded as 3 (strong positive), with lower levels of expression graded as 2 (positive) or 1 (weak positive), and no detectable expression graded as 0 (negative). Proportions of positive cells were graded as 0 (0-5\% positive), 1 (6-25\% positive), 2 (26-50\% positive) or 3 ( $>50 \%$ positive). The scores for staining intensity and proportion of positive cells were multiplied to produce a weighted score for each case. A mean value was calculated when heterogeneity was present in the three separate TMA cores. Different cut-off values were investigated in the study and the best ones selected. For KDM6A, the complete absence of nuclear staining within tumor cells was considered negative. For EZH 2 and H3K27me3, cases with weighted scores of $\leq 1$ were defined as negative. All other scores were considered positive.

Statistical analysis. All data processing and statistical analyses were performed using SPSS software (version 16.0; SPSS, Inc.). Pearson's $\chi^{2}$ test and Fisher's exact test were used to analyze the associations between the IHC staining results and the clinicopathological parameters. The 3-year survival rates were calculated using the Kaplan-Meier method. CSS and DFS analyses were performed using the Kaplan-Meier method and log-rank test. Multivariate predictive modeling was performed using multivariate Cox regression analysis. Qualifying criteria for inclusion in the Cox regression analysis were a P-value of $\leq 0.1$ or a risk ratio of $\leq 0.5$ or $>2$ on univariate Cox analysis. For the aforementioned statistical tests, $\mathrm{P}<0.05$ was used to indicate a statistically significant difference. McNemar's test and Bonferroni's correction were used to analyze the association between the expression levels of any two out of the three proteins; a P-value $<0.0167$ (the P-value equals 0.05 divided by 3 ) represented a significant difference.

\section{Results}

Patient characteristics. UTUC specimens were obtained from 108 patients with a male-to-female ratio of 1.35:1. The median age of the patients at diagnosis was 70 (range, 41-86 years). Tumor grade was low in 31 patients and high in 77 patients. Low grade means the tumor is composed of slender papillary structure with common papillary branches and fusions and increased epithelial layers. The cell abnormalities are not obvious under low magnification. While high grade means the coated epithelium showed obvious disorder of arrangement and cell atypia. Characteristically, there is striking nuclear 
Table I. Summary of the clinicopathological characteristics of patients with upper tract urothelial carcinoma $(n=108)$.

\begin{tabular}{lll}
\hline Characteristics & $\mathrm{n}$ & $\%$ \\
\hline
\end{tabular}

\section{Patient age, years}

$<65$

$\geq 65$

Sex

Male

Female

Laterality

Left

Right

Both

Tumor site

Renal pelvis

Ureter

Transitional zone

Renal pelvis and ureter

Tumor size, cm

$<3.0$

$\geq 3.0$

Tumor grade

Low

High

Pathological stage

NMI (pTa + pT1)

pTa

pT1

$\mathrm{MI}(\mathrm{pT} 2+\mathrm{pT} 3+\mathrm{pT} 4)$

pT2

pT3

pT4

LVI

No

Yes

Neural invasion

No

Yes

Lymph node metastasis

No

Yes

Concurrent CIS

No

Yes

Extensive necrosis

No

Yes

Glomerular sclerosis

No

Yes

Solitary or multifocality

Solitary

Multifocality
40

68

37.0

63.0

57.4

42.6

45.4

53.7

0.9

38.9

50.9

9.3

0.9

55.6

44.4

28.7

71.3

39.8

30.6

9.3

60.2

24.1

28.7

7.4

80.6

19.4

91.7

90.7

86.1

13.9

84.3

15.7

67.6

32.4

86.1
Table I. Continued.

\begin{tabular}{lrr}
\hline Characteristics & $\mathrm{n}$ & $\%$ \\
\hline Bladder cancer $^{\mathrm{a}}$ & & \\
No & 61 & 72.6 \\
Simultaneous & 5 & 6.0 \\
Postoperative & 16 & 19.0 \\
Preoperative & 2 & 2.4 \\
Status ${ }^{\text {b,c }}$ & & \\
Survival & 66 & 70.2 \\
Death & 28 & 29.8 \\
Cancer-specific survival, years & \\
$<1$ & & \\
1-3 & 19 & 20.2 \\
$>3$ & 41 & 43.6 \\
Disease-free survival, years & & \\
$<1$ & 34 & 36.2 \\
1-3 & & \\
$>3$ & 36 & 38.3 \\
\hline
\end{tabular}

${ }^{\mathrm{a}}$ Clinical information on bladder cancer recurrence was obtained in 84 patients; ${ }^{b}$ median follow-up time, 28 months; ${ }^{c}$ complete follow-up information was obtained in 94 patients. NMI, non-muscle invasive; pT, pathological tumor; LVI, lymphovascular invasion; CIS, carcinoma in situ.

pleomorphism with variably sized and shaped hyperchromatic nuclei. Mitoses are numerous and abnormal mitotic figures are present. The prognosis of patients with muscle-invasive urothelial carcinoma was worse than the patients with non-muscle invasive ones and the clinical protocol was different for these two groups $(4,14)$. Thus, a total of 43 patients with pathological stage pTa-pT1 were categorized as the non-muscle invasive group, while 65 patients with stage pT2-pT4 were categorized as the muscle invasive group. During the observation period, complete follow-up information was obtained for 94 patients and included 28 patients $(29.8 \%)$ who died of tumor progression.

The data from all 108 patients were analyzed and only those with follow-up data were included in the analysis of prognosis. The clinicopathological characteristics of these patients with UTUC are summarized in Table I.

Negative KDM6A expression status is associated with aggressive clinical behavior in UTUC. Immunohistochemical staining of KDM6A expression in the TMAs is shown in Fig. 1 and Fig. S1. Compared with that in normal urothelial cells, the expression of KDM6A was significantly lower in tumor cells (79.3 vs. $27.6 \%$; $\mathrm{P}=0.033$ ) (Table $\mathrm{SI}$ ).

The association between KDM6A protein expression level and patient clinicopathological parameters was also analyzed (Table II). Low KDM6A expression was associated with larger tumor size $(36 / 48$ vs. $34 / 60 ; \mathrm{P}=0.047)$, higher tumor grade $(56 / 77$ vs. $14 / 31 ; \mathrm{P}=0.007)$ and advanced pathological stage (48/65 vs. $22 / 43 ; \mathrm{P}=0.016)$. There was no association between KDM6A expression level and the other parameters. 

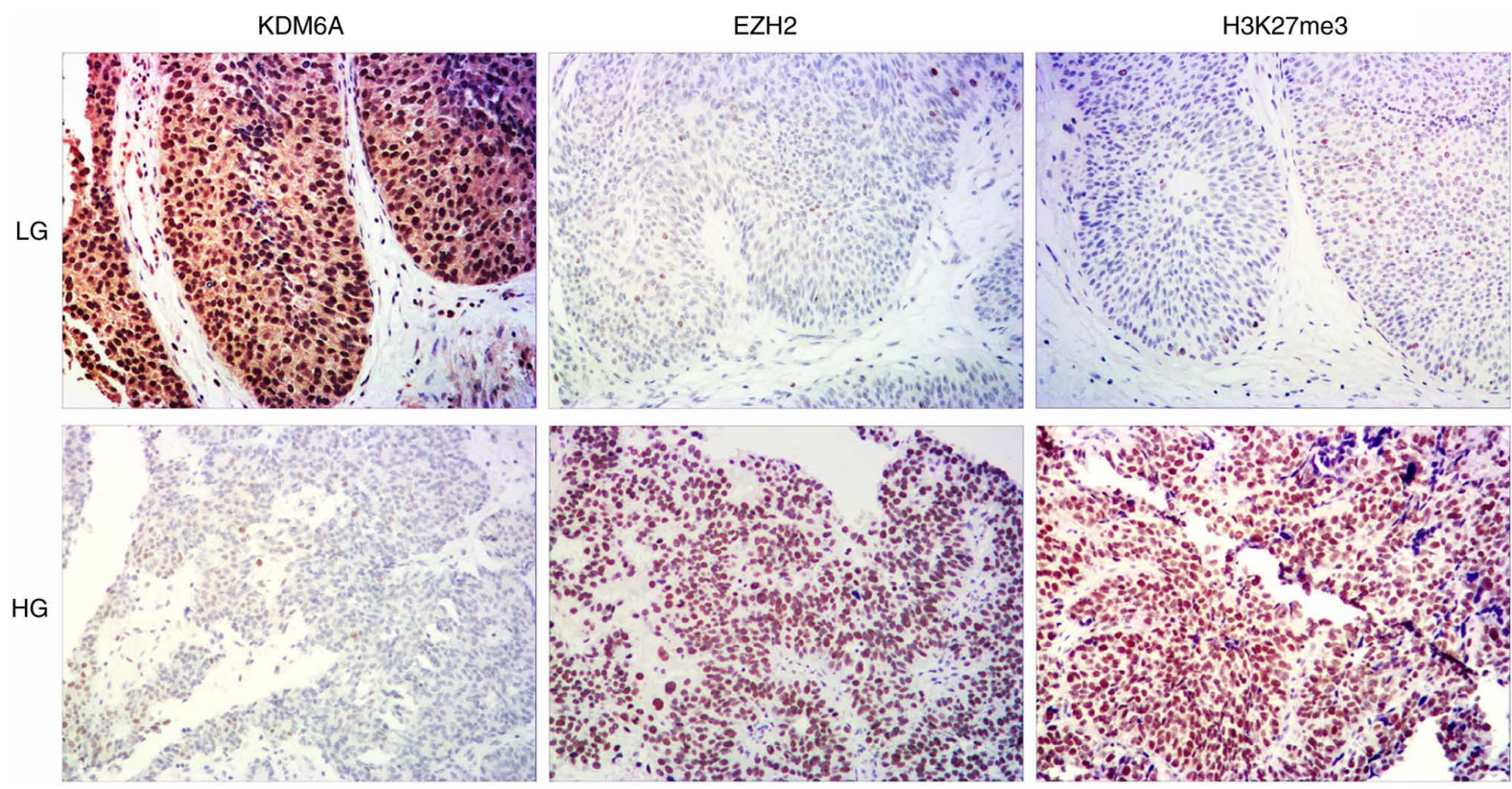

Figure 1. Representative images of staining for KDM6A, EZH2 and H3K27me3 in LG and HG urothelial carcinoma. Magnification x100. KDM6A, lysine demethylase 6A; EZH2, histone-lysine N-methyltransferase EZH2; LG, low grade; HG, high grade.

A

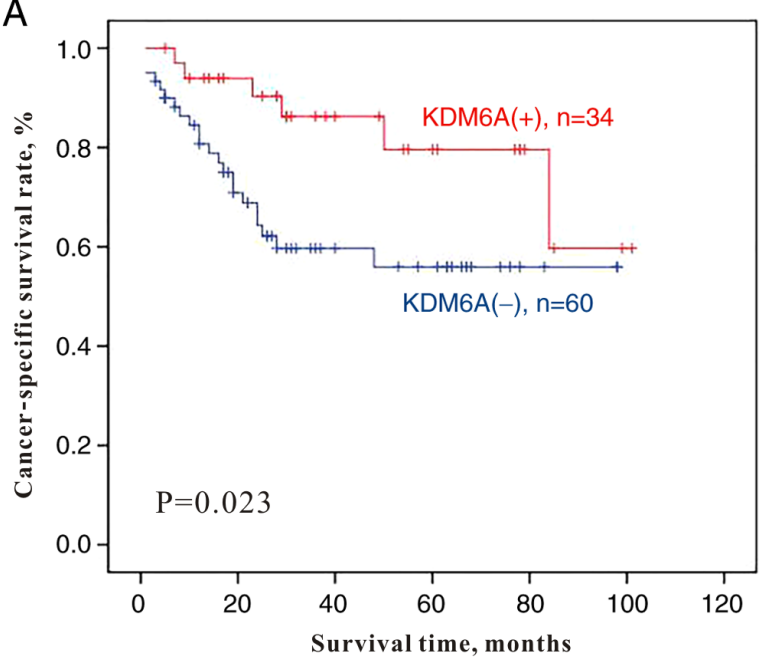

B

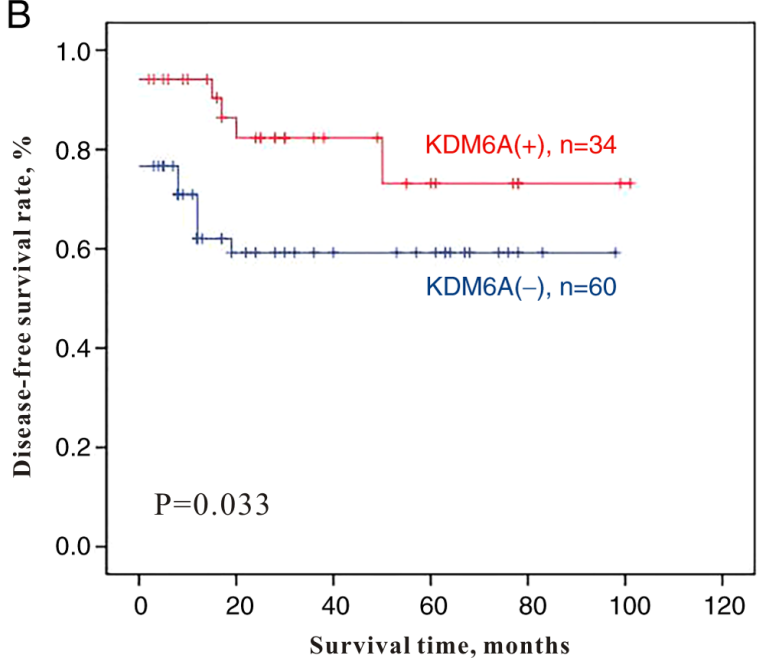

Figure 2. Survival curves using the Kaplan-Meier method with the log-rank test. (A) Patient cancer-specific survival. (B) Patient disease-free survival. KDM6A, lysine demethylase 6A.

Lower KDM6A expression level is a useful prognostic factor for poor prognosis in patients with UTUC. Of the 94 patients with complete follow-up information, 66 patients $(70.2 \%)$ were alive at a median follow-up time of 35.5 months (range, 3-101 months). A total of 28 (29.8\%) patients died of the disease following a median period of 15 months (range, 1-84 months). Kaplan-Meier single-factor analysis and the log-rank test demonstrated a statistically significant decrease in CSS $(\mathrm{P}=0.023)$ and DFS $(\mathrm{P}=0.033)$ times in patients with negative staining for KDM6A (Table III; Fig. 2). However, KDM6A was not an independent risk factor for either CSS or DFS according to multivariate Cox regression analysis. As expected, other adverse clinicopathological parameters, such as high tumor grade, advanced stage, presence of lymphovascular invasion and lymph node metastasis were associated with both shorter CSS $(\mathrm{P}=0.036, \mathrm{P}<0.001, \mathrm{P}=0.030$ and $\mathrm{P}=0.002$, respectively) and DFS ( $\mathrm{P}=0.041, \mathrm{P}<0.001, \mathrm{P}=0.031$ and $\mathrm{P}=0.008$, respectively) times. Furthermore, only advanced stage was an independent prognostic factor for both CSS and DFS times ( $\mathrm{P}=0.013$ and $\mathrm{P}=0.015$, respectively).

Lower KDM6A expression level is neither associated with a significant increase in $\mathrm{H} 3 \mathrm{~K} 27 \mathrm{me} 3$ expression nor with EZH2 expression. To evaluate the association between KDM6A and $\mathrm{EZH} 2$, and $\mathrm{H} 3 \mathrm{~K} 27 \mathrm{me} 3$ expression levels in UTUC, the expression levels of EZH2 and H3K27me3 in UTUC and the adjacent normal urothelium were investigated. There was a significant increase in EZH2 expression level in UTUC tumors compared with that in the normal urothelium $(\mathrm{P}=0.035$; Table $\mathrm{SI})$. However, H3K27me3 expression levels were similar between 
Table II. Clinicopathological characteristics associated with KDM6A, EZH2 and H3K27me3 expression.

\begin{tabular}{|c|c|c|c|c|c|c|c|c|c|}
\hline \multirow[b]{2}{*}{ Characteristic } & \multicolumn{3}{|c|}{ KDM6A } & \multicolumn{3}{|c|}{ EZH2 } & \multicolumn{3}{|c|}{ H3K27me3 } \\
\hline &,$- \mathrm{n}$ &,$+ \mathrm{n}$ & P-value &,$- \mathrm{n}$ &,$+ \mathrm{n}$ & P-value &,$- \mathrm{n}$ &,$+ \mathrm{n}$ & P-value \\
\hline Patient age, years & & & 0.654 & & & 0.516 & & & 0.883 \\
\hline$<65$ & 27 & 13 & & 18 & 22 & & 20 & 20 & \\
\hline$\geq 65$ & 43 & 25 & & 35 & 33 & & 35 & 33 & \\
\hline Sex & & & 1.000 & & & 0.316 & & & 0.868 \\
\hline Male & 40 & 22 & & 33 & 29 & & 32 & 30 & \\
\hline Female & 30 & 16 & & 20 & 26 & & 23 & 23 & \\
\hline Laterality & & & 0.122 & & & 0.053 & & & 0.122 \\
\hline Left & 36 & 13 & & 29 & 20 & & 21 & 28 & \\
\hline Right & 33 & 25 & & 23 & 35 & & 34 & 24 & \\
\hline Both & 1 & 0 & & 1 & 0 & & 0 & 1 & \\
\hline Tumor site & & & 0.082 & & & 0.319 & & & \\
\hline Renal pelvis & 32 & 10 & & 24 & 18 & & 24 & 18 & 0.518 \\
\hline Ureter & 31 & 24 & & 23 & 32 & & 26 & 29 & \\
\hline Transitional zone & 7 & 3 & & 5 & 5 & & 4 & 6 & \\
\hline Renal pelvis and ureter & 0 & 1 & & 1 & 0 & & 1 & 0 & \\
\hline Tumor size, $\mathrm{cm}$ & & & 0.047 & & & 0.863 & & & 0.344 \\
\hline$<3.0$ & 34 & 26 & & 29 & 31 & & 33 & 27 & \\
\hline$\geq 3.0$ & 36 & 12 & & 24 & 24 & & 22 & 26 & \\
\hline Tumor grade & & & 0.007 & & & 0.001 & & & 0.073 \\
\hline Low & 14 & 17 & & 23 & 8 & & 20 & 11 & \\
\hline High & 56 & 21 & & 30 & 47 & & 35 & 42 & \\
\hline Pathological stage & & & 0.016 & & & 0.002 & & & 0.223 \\
\hline NMI (pTa-pT1) & 22 & 21 & & 29 & 14 & & 25 & 18 & \\
\hline MI (pT2-pT4) & 48 & 17 & & 24 & 41 & & 30 & 35 & \\
\hline LVI & & & 0.126 & & & 0.882 & & & 0.736 \\
\hline No & 53 & 34 & & 43 & 44 & & 45 & 42 & \\
\hline Yes & 17 & 4 & & 10 & 11 & & 10 & 11 & \\
\hline Neural invasion & & & 1.000 & & & 0.162 & & & 0.316 \\
\hline No & 64 & 35 & & 51 & 48 & & 52 & 47 & \\
\hline Yes & 6 & 3 & & 2 & 7 & & 3 & 6 & \\
\hline Lymph node metastasis & & & 0.739 & & & 0.951 & & & 0.742 \\
\hline No & 64 & 34 & & 48 & 50 & & 49 & 49 & \\
\hline Yes & 6 & 4 & & 5 & 5 & & 6 & 4 & \\
\hline Concurrent CIS & & & 0.674 & & & 0.094 & & & 0.362 \\
\hline No & 61 & 32 & & 49 & 44 & & 49 & 44 & \\
\hline Yes & 9 & 6 & & 4 & 11 & & 6 & 9 & \\
\hline Extensive necrosis & & & 0.408 & & & 0.033 & & & 0.856 \\
\hline No & 57 & 34 & & 49 & 42 & & 46 & 45 & \\
\hline Yes & 13 & 4 & & 4 & 13 & & 9 & 8 & \\
\hline Glomerular sclerosis & & & 0.892 & & & 0.735 & & & 0.192 \\
\hline No & 47 & 26 & & 35 & 38 & & 34 & 39 & \\
\hline Yes & 23 & 12 & & 18 & 17 & & 21 & 14 & \\
\hline Solitary or multifocality & & & 0.249 & & & 0.094 & & & 0.142 \\
\hline Solitary & 58 & 35 & & 49 & 44 & & 50 & 43 & \\
\hline Multifocality & 12 & 3 & & 4 & 11 & & 5 & 10 & \\
\hline Bladder cancer & & & 0.842 & & & 0.116 & & & 0.701 \\
\hline No & 35 & 26 & & 29 & 32 & & 32 & 29 & \\
\hline Simultaneous & 3 & 2 & & 5 & 0 & & 3 & 2 & \\
\hline Postoperative & 10 & 6 & & 9 & 7 & & 11 & 5 & \\
\hline Preoperative & 2 & 0 & & 1 & 1 & & 1 & 1 & \\
\hline
\end{tabular}

NMI, non-muscle invasive; pT, pathological tumor; LVI, lymphovascular invasion; CIS, carcinoma in situ; KDM6A, lysine demethylase 6A; EZH2, histone-lysine N-methyltransferase EZH2. 


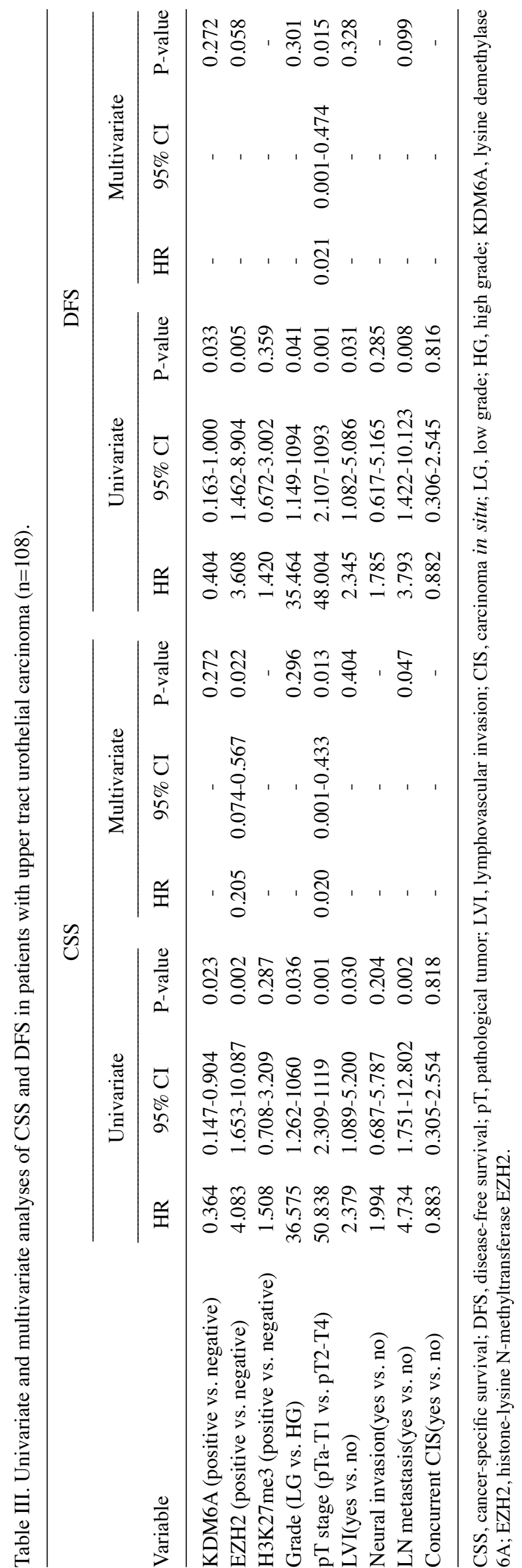

Table IV. Pairwise association among the KDM6A, EZH2 and $\mathrm{H} 3 \mathrm{~K} 27 \mathrm{me} 3$ expression levels in upper tract urothelial carcinoma.

\begin{tabular}{|c|c|c|c|c|}
\hline \multirow[b]{2}{*}{ Expression } & \multirow[b]{2}{*}{$\mathrm{n}$} & \multicolumn{2}{|c|}{ KDM6A } & \multirow[b]{2}{*}{ P-value ${ }^{b}$} \\
\hline & & $+($ cutoff 0$), n$ & P-value ${ }^{a}$ & \\
\hline EZH2- & 53 & 21 & 0.037 & 0.871 \\
\hline $\mathrm{EZH}^{+}{ }^{+}$ & 55 & 17 & & \\
\hline H3K27me3- & 55 & 27 & 0.092 & \\
\hline $\mathrm{H} 3 \mathrm{~K} 27 \mathrm{me}^{+}$ & 53 & 11 & & \\
\hline
\end{tabular}

${ }^{\mathrm{a}}$ Association between positive and negative expression; ${ }^{\mathrm{b}}$ association between EZH2 and H3K27me3 expression levels. KDM6A, lysine demethylase 6A; EZH2, histone-lysine N-methyltransferase EZH2.

UTUC tumor and normal urothelium samples $(\mathrm{P}=0.390$; Table SI). In view of the fact that KDM6A antagonizes $E Z H 2$ activity and functions as a histone $\mathrm{H} 3 \mathrm{~K} 27 \mathrm{me} 3$ demethylase, pairwise associations among the expression levels of these three proteins were also analyzed. However, there was no statistical association observed (Table IV).

The clinicopathological significance of EZH2 and H3K27me3 expression levels was also analyzed (Table II). There was an association between EZH2 expression levels and tumor grade $(\mathrm{P}=0.001)$, advanced pathological stage $(\mathrm{P}=0.002)$ and extensive necrosis $(\mathrm{P}=0.033)$. Using multivariate survival analysis, increased EZH2 expression levels were a significant predictor for poor CSS time $(\mathrm{P}=0.022)$, while there was no significance for DFS time ( $\mathrm{P}=0.058)$ (Table III). Upon analyzing only $\mathrm{H} 3 \mathrm{~K} 27 \mathrm{me} 3$ protein expression, its level was neither significantly associated with any clinicopathological parameter nor was it of prognostic value.

\section{Discussion}

A previous study has shown that KDM6A is the most frequently mutated gene in AA-associated UTUCs, and this type of cancer was found to be the most common UTUC in a Chinese cohort in one study (3). However, currently there is a lack of relevant research with respect to KDM6A expression in patients with UTUC in China. To the best of our knowledge, this is the first study in which the association between KDM6A expression levels and clinicopathologically relevant features of UTUC has been evaluated.

KDM6A mutations have been reported to be involved in both hematological and solid neoplasms, including multiple myeloma, clear cell renal cell carcinoma, esophageal squamous cell carcinoma and bladder cancer (5,15-18); however, the expression levels of KDM6A vary in the different tumor types. Inactivating mutations and downregulation of KDM6A have been described in a wide spectrum of bladder urothelial carcinomas (4). Conversely, in breast cancer and clear cell renal cell carcinoma, KDM6A overexpression and upregulation has been associated with a poor prognosis (19-21). These varying results suggest that the epigenetic function of KDM6A in human cancer may depend on a number of factors, including the type of cell involved in the cancer. In the present study, 
KDM6A expression was significantly lower in Chinese patients with UTUC compared with that in adjacent normal tissues, with adverse pathological features and an unfavorable prognosis, which suggested that lower KDM6A expression may accelerate UTUC progression.

Patients with UTUC in China were associated with specific epidemiological characteristics (2). An observed epidemiological difference between patients with UTUC in China and Western countries is sex distribution (2). In Western countries, urothelial carcinoma is more common in men than in women (2). A previous study revealed that the $\mathrm{X}$ chromosome protects against bladder cancer among women, via a KDM6A-dependent epigenetic mechanism, and that decreased expression of KDM6A predicts a poor prognosis in women with bladder cancer (22). This suggests that KDM6A could be a prototypical sex-biasing tumor suppressor (22). By contrast, the incidence rate of UTUC in China is higher in women compared with that in men (2). However, in the present study, it was found that KDM6A was neither significantly associated with sex or with the survival time of female patients with UTUC. Therefore, the mechanisms underlying sex differences of urothelial carcinoma associated with KDM6A, and the extent to which KDM6A contributes to sex differences in Chinese patients with UTUC warrant further research.

Geographic differences in risk factors for UTUC, particularly AA consumption, may explain some differences in the epidemiological and clinicopathological characteristics of patients with UTUC in China and Western countries (3). Notably, high-throughput genome-wide screening identified KDM6A as the most frequently mutated gene in AA-associated UTUC (3). However, patient information regarding AA usage was not available in the present retrospective study, as it was difficult to trace back the patient history of medication with Chinese herbs, which take decades to cause nephrotoxicity and UTUC. Therefore, prospective studies will be required to investigate the role of KDM6A in Chinese patients with UTUC, particularly in AA-associated UTUC.

Previous studies have shown that homeostasis of $\mathrm{H} 3 \mathrm{~K} 27 \mathrm{me} 3$ levels is maintained by coordination of the demethylase KDM6A and PRC2 complex, containing methyltransferase $\mathrm{EZH} 2(4,23)$, and that this balance is disrupted when KDM6A is mutated. The association between KDM6A and EZH2, as investigated in different studies on various types of carcinoma, remains unclear. For example, in human lung cancer, KDM6A expression was shown to be inversely correlated with EZH2 expression (24). However, a study of chronic myelomonocytic leukemia identified loss-of-function mutations in both KDM6A and EZH2 despite their opposing roles in H3K27me3 regulation (25). In bladder cancer, the levels of EZH2 expression were found to be similar in different $K D M 6 A$ gene status (4). Consistent with the findings in bladder cancer, no statistical correlation between KDM6A and EZH2 expression levels was found. A possible explanation is that KDM6A may antagonize $E Z H 2$ activity at the genetic level, but is not associated with $\mathrm{EZH} 2$ at the protein expression level. Aside from the enzymatic subunit of EZH2, the PRC2 complex also contains other cofactors, such as SUZ12, EZH1 and EED (26). It would therefore be of interest to investigate the expression level of all the other core PRC components independent of EZH2 status in future studies.
The present study has some limitations. The study is retrospective in nature, and the materials used were paraffin-embedded tissues, which were not suitable for western blotting or reverse transcription quantitative-PCR analysis. Further investigations of the mechanism of KDM6A in UTUC progression will therefore be analyzed using fresh UTUC tumor tissues and cell lines in the next project.

In conclusion, a lower KDM6A expression level was associated with adverse pathological features, such as a high grade and advanced stage of UTUC, and was a significant predictor of a poor prognosis in patients with UTUC. These data suggested that immunohistochemical analysis of KDM6A provides an easier and more efficient method, compared with complex genome screening procedures used in other studies $(3,4)$, for predicting the prognosis in patients with UTUC in a clinical setting. KDM6A expression level was not associated with EZH2 or H3K27me3 expression levels. Therefore, lower KDM6A expression may not confer sensitivity to either EZH2 inhibition or EZH2 inhibitor-based therapy and remains to be further investigated.

\section{Acknowledgements}

The authors would like to thank Dr Lei Wang (Department of Urology, Peking University Wu Jieping Urology Center, Peking University Shougang Hospital, Beijing, China) for providing suggestions on the study design.

\section{Funding}

The present study was funded by the Leading Academic Discipline Project of Beijing Education Bureau (grant no. BMU20110254) and the Peking University Shougang Hospital Youth Foundation (grant no. SGYYQ201701).

\section{Availability of data and materials}

The datasets analyzed during the present study are available from the corresponding author on reasonable request.

\section{Authors' contributions}

YW and HYH designed the study and performed the experiments, SFW and YZ collected and interpreted the immunohistochemistry data, YW and JXZ analyzed the data, and YW and HYH prepared the manuscript. All authors read and approved the final manuscript.

\section{Ethics approval and consent to participate}

The present study was performed according to the principles of the Helsinki declaration. Experimental protocols were reviewed and approved by the Ethics Committee of the Peking University Shougang Hospital (approval no. IRBK-2017-047-01). Written informed consent was obtained from all patients.

\section{Patient consent for publication}

Not applicable. 


\section{Competing interests}

The authors declare that they have no competing interests.

\section{References}

1. Chen XP, Xiong GY, Li XS, Matin SF, Garcia M, Fang D, Wang TY, Yu W, Gong K, Song Y, et al: Predictive factors for worse pathological outcomes of upper tract urothelial carcinoma: Experience from a nationwide high-volume centre in China. BJU Int 112: 917-924, 2013.

2. Singla N, Fang D, Su X, Bao Z, Cao Z, Jafri SM, Xiong G, Zhang L, Hutchinson R, Sagalowsky A, et al: A multi-institutional comparison of clinicopathologic characteristics and oncologic outcomes of upper tract urothelial carcinoma in China and the United States. J Urol 197:1208-1213, 2016.

3. Poon SL, Pang ST, McPherson JR, Yu W, Huang KK, Guan P, Weng WH, Siew EY, Liu Y, Heng HL, et al: Genome-wide mutational signatures of aristolochic acid and its application as a screening tool. Sci Transl Med 5: 197ra101, 2013.

4. Ler LD, Ghosh S, Chai X, Thike AA, Heng HL, Siew EY, Dey S, Koh LK, Lim JQ, Lim WK, et al: Loss of tumor suppressor KDM6A amplifies PRC2-regulated transcriptional repression in bladder cancer and can be targeted through inhibition of EZH2. Sci Transl Med 9: eaai8312, 2017.

5. Van der Meulen J, Speleman F and Van Vlierberghe P: The H3K 27me3 demethylase UTX in normal development and disease. Epigenetics 9: 658-668, 2014.

6. Nickerson ML, Dancik GM, Im KM, Edwards MG, Turan S, Brown J, Ruiz-Rodriguez C, Owens C, Costello JC, Guo G, et al: Concurrent alterations in TERT, KDM6A, and the BRCA pathway in bladder cancer. Clin Cancer Res 20: 4935-4948, 2014.

7. Grasso CS, Wu YM, Robinson DR, Cao X, Dhanasekaran SM, Khan AP, Quist MJ, Jing X, Lonigro RJ, Brenner JC, et al: The mutational landscape of lethal castrate resistant prostate cancer. Nature 487: 239-243, 2012.

8. Kandoth C, McLellan MD, Vandin F, Ye K, Niu B, Lu C, Xie M, Zhang Q, McMichael JF, Wyczalkowski MA, et al: Mutational landscape and significance across 12 major cancer types. Nature 502: 333-339, 2013.

9. Lan F, Bayliss PE, Rinn JL, Whetstine JR, Wang JK, Chen S, Iwase S, Alpatov R, Issaeva I, Canaani E, et al: A histone H3 lysine 27 demethylase regulates animal posterior development. Nature 449: 689-694, 2007.

10. Hong S, Cho YW, Yu LR, Yu H, Veenstra TD and Ge K: Identification of JmjC domain-containing UTX and JMJD3 as histone H3 lysine 27 demethylases. Proc Natl Acad Sci USA 104: 18439-18444, 2007.

11. Moch H, Humphrey PA, Ulbright TM, Cubilla AL and Reuter VE: WHO Classification of Tumours of the Urinary System and Male Genital Organ. International Agency for Research on Cancer (IARC), Lyon, p131, 2016.
12. Paner GP, Stadler WM, Hansel DE, Montironi R, Lin DW and Amin MB: Updates in the eighth edition of the Tumor-Node-Metastasis staging classification for urologic cancers. Eur Uro 173: 560-569, 2018.

13. Rubin MA, Dunn R, Strawderman M and Pienta KJ: Tissue microarray sampling strategy for prostate cancer biomarker analysis. Am J Surg Pathol 26: 312-319, 2002.

14. Li YR, Yu KJ, Chang YH, Lin PH, Shao IH, Kan HC, Chu YC, Chuang CK, Pang ST and Liu CY: Predictors of intravesical recurrence after radical nephroureterectomy and prognosis in patients with upper tract urothelial carcinoma. Cancer Manag Res 12: 7439-7450, 2020.

15. Gossage L, Murtaza M, Slatter AF, Lichtenstein CP, Warren A, Haynes B, Marass F, Roberts I, Shanahan SJ, Claas A, et al: Clinical and pathological impact of VHL, PBRMI, BAPI, SETD2, KDM6A, and JARIDIc in clear cell renal cell carcinoma. Genes Chromosomes Cancer 53: 38-51, 2014.

16. Ntziachiristos P, Tsirigos A, Welstead GG, Trimarchi T, Bakogianni S, Xu L, Loizou E, Holmfeldt L, Strikoudis A, King B, et al: Contrasting roles for histone 3 lysine 27 demethylases in acute lymphoblastic leukemia. Nature 514: 513-517, 2014.

17. Bailey P, Chang DK, Nones K, Johns AL, Patch AM, Gingras MC, Miller DK, Christ AN, Bruxner TJC, Quinn MC, et al: Genomic analyses identify molecular subtypes if pancreatic cancer. Nature 531: 47-52, 2016

18. Li SH, Lu HI, Huang WT, Tien WY, Lan YC, Lin WC, Tsai HT and Chen CH: The prognostic significance of histone demethylase UTX in esophageal squamous cell carcinoma. Int J Mol Sci 19: 297, 2018.

19. Paolicchi E, Crea F, Farrar WL, Green JE and Danesi R: Histone lysine demethylases in breast cancer. Crit Rev Oncol Hematol 86: 97-103, 2013.

20. Curtis C, Shah SP, Chin SF, Turashvili G, Rueda OM, Dunning MJ, Speed D, Lynch AG, Samarajiwa S, Yuan Y, et al: The genomic and transcriptomic architecture of 2,000 breast tumours reveals novel subgroups. Nature 486: 346-352, 2012.

21. Wang J, Liu L, Xi W, Long Q, Wang Y, Bai Q, Xia Y, Xu J and Guo J: Prognostic value of UTX expression in patients with clear cell renal cell carcinoma. Urol Oncol 34: 338.e19-27, 2016.

22. Kaneko S and Li X: X chromosome protects against bladder cancer in females via a KDM6A-dependent epigenetic mechanism. Sci Adv 4: eaar5598, 2018.

23. Martinez-Garcia E and Licht JD: Deregulation of H3K27 methylation in cancer. Nat Genet 42: 100-101, 2010.

24. Wu Q, Tian Y, Zhang J, Tong X, Huang H, Li S, Zhao H, Tang Y, Yuan C, Wang K, et al: In vivo CRISPR screening unveils histone demethylase UTX as an important epigenetic regulator in lung tumorigenesis. Proc Natl Acad Sci USA 115: E3978-E3986, 2018.

25. Jankowska AM, Makishima H, Tiu RV, Szpurka H, Huang Y, Traina F, Visconte V, Sugimoto Y, Prince C, O'Keefe C, et al: Mutational spectrum analysis of chronic myelomonocytic leukemia includes genes associated with epigenetic regulation: UTX, EZH2, and DNMT3A. Blood 118: 3932-3941, 2011.

26. Kim KH and Roberts CW: Targeting EZH2 in cancer. Nat Med 22: 128-134, 2016. 